\title{
Characterizing Unlayered-Graphene in Homemade Core\Rim Carbon Raindrops
}

\author{
Tristan Hundley ${ }^{1}$ and P. Fraundorf ${ }^{1,2}$ \\ 1. Physics \& Astronomy/Center for Nanoscience Bldg., U. Missouri-SL, St. Louis, USA. \\ 2. Physics Department, Washington University, St. Louis, USA.
}

A significant fraction of micron-sized presolar "high-density" graphite onions (e.g. from Murchison KFC1) likely formed in AGB star atmospheres[1], and have concentric graphite shells surrounding a core of unlayered graphene $[2,3]$. HR-TEM images suggest flat $4[\mathrm{~nm}]$ wide graphene sheets connected by pentagonal defects [4]. The absence of "carbide seeds" sometimes found in presolar onions without these cores, plus their "almost graphitic" densities [5] and spherical shapes with a sharp transition to a graphitic rim, all suggest homogeneous nucleation and atom-by-atom growth from the vapor phase onto a super-cooled liquid droplet, followed by graphite rim growth after droplet solidification [6,7].

The unlayered-graphene core of these micron-sized particles has been easiest to characterize via TEM when microtomed (Fig. 1a), although it has been possible get diffraction contrast from the graphite rims in unsliced particles less than half micron in diameter (Fig. 1b). In order to examine the conditions under which this unlayered-graphene forms, and the information contained in the (e.g. $4 \mathrm{~nm})$ coherence widths of the graphene sheets and phase-contrast images suggesting a faceted-pentacone mode of nucleation and growth, we've attempted to "slow cool" carbon vapor in the interior of a resistively-heated and evaporating graphite oven [10] here on earth.

Sample particles from anneals with $6.2 \mathrm{~mm}$ diameter hollow graphite ovens, resistively heated in a Balzer's turbopumped vacuum evaporator, are shown in Fig. 2. Exterior color temperatures maps were estimated with Nikon 1 camera images of the oven seen through neutral density filters, and well as cellphone camera "movies". Particles were collected using a Pt loop from deposited material on a "flat" inside the oven cavity, and examined on holey or lacey carbon supports in an EM430ST TEM. The particles (cf. Fig. 2) include core-rim onions, and some rim-only graphite onions, like those seen in the presolar collections. Preliminary evidence also suggests that we may be finding some core-only particles.

For the unsliced particles (typically a quarter micron in diameter) we can get diffraction rings from (hk0) spacings which confirm "random-layer lattice" configurations (as expected) for the graphene sheets in the rim, but of course in transmission these will always interfere with the (hk0) spacings from the core material itself. Quantitative diffraction analysis using rim/core particle models is one path for exploring these "homemade" carbon droplets further, both for comparison to presolar particle data to help us understand the formation of carbon raindrops in the atmosphere of "cool" giant stars, and for learning to synthesize unlayered graphene and explore its promise as a general purpose diffusion barrier. HRTEM work will likely require core regions that have been torn after slicing [8].

\section{References:}

[1] Amari et al, LPS XLII (2011), Abstract \#1098.

[2] Bernatowicz et al, Astrophys. J. 472 (1996), p. 760.

[3] T. K. Croat, Elements 10 (2014), p. 441.

[4] Fraundorf and Wackenhut, Astrophys. J. Lett. 578 (2002), L153-L156. 
[5] P. Fraundorf et al, Microscopy \& Microanalysis 16:2 (2010), p. 1534.

[6] P. Fraundorf et al, Microscopy \& Microanalysis 22:S3 (2016), p. 1816, HAL-01356394.

[7] M. Lipp et al, Microscopy \& Microanalysis 23:S1 (2017), p. 2192.

[8]. Thanks to Roy Lewis of U. Chicago for providing these meteorite dissolution residues, and for Tom Bernatowicz of Washington U. in St. Louis for the presolar grain microtome work.

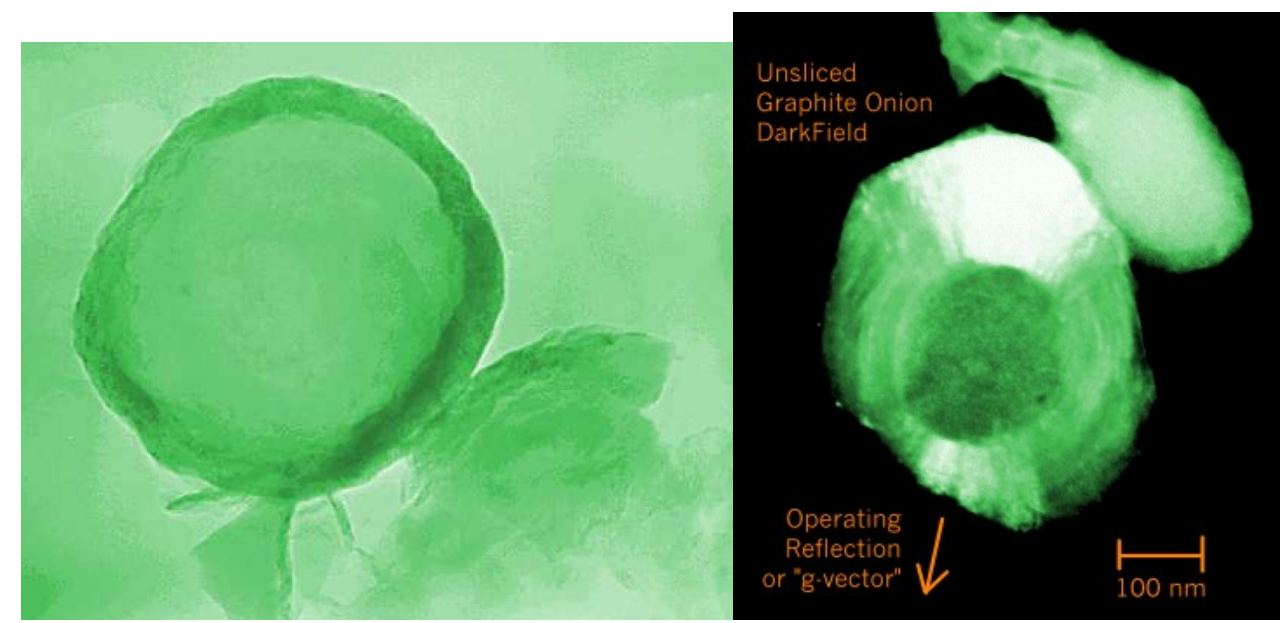

Figure 1. Brightfield TEM image of a sliced 0.9 micron diameter presolar core rim onion (left), and darkfield image of an unsliced one, both extracted from the Murchison Meteorite.

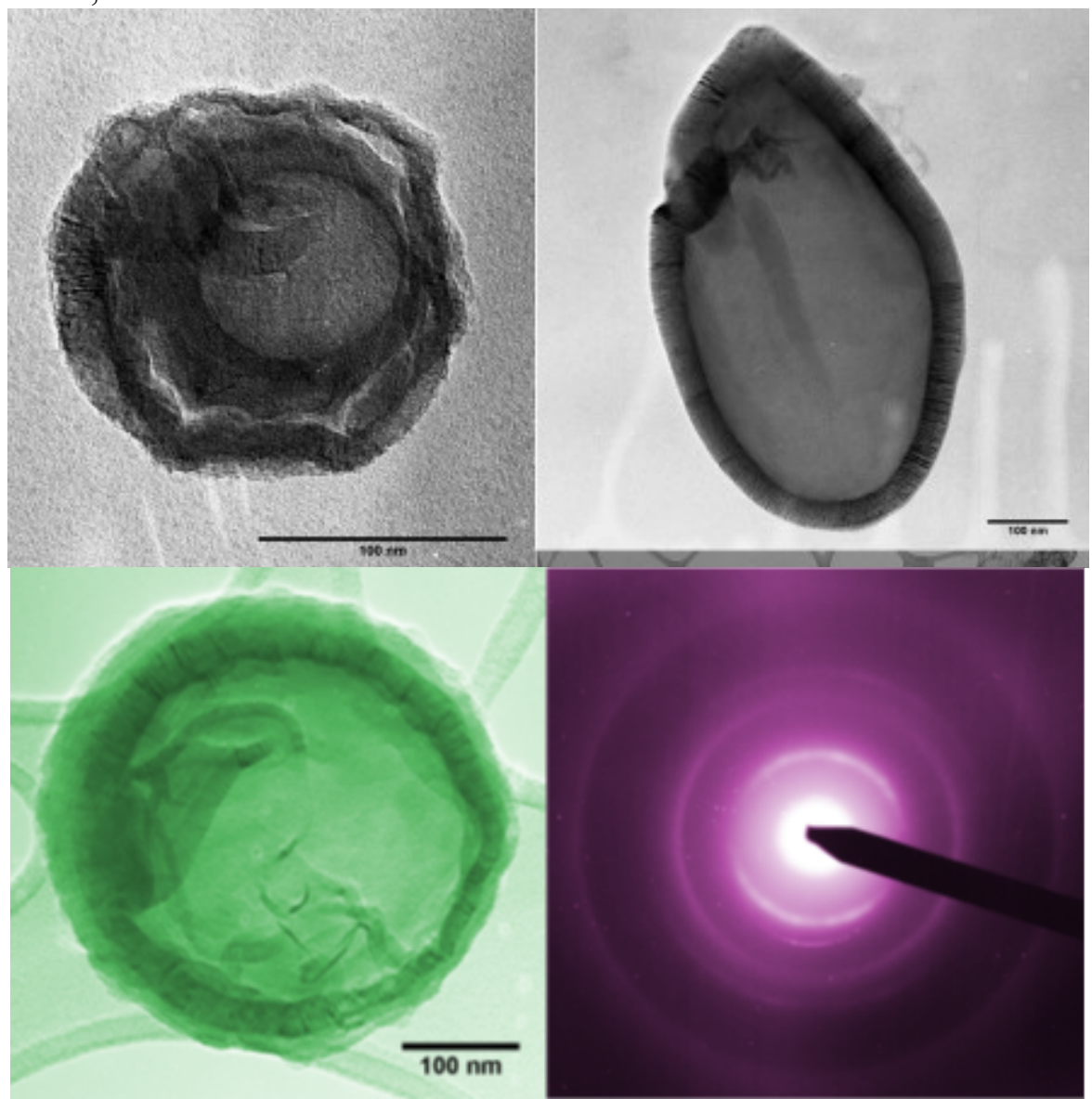

Figure 2. At top and bottom left are "homemade" unsliced core-rim onions, one small, one oval in shape, and the third quite spherical. At bottom right is an SAED pattern of the whole particle. 